\title{
EMPRESARIOS MIGRANTES MEXICANOS EN ESTADOS UNIDOS
}

\section{EMPLOYERS MEXICAN MIGRANTS IN THE UNITED STATES}

Eduardo Fernández Guzmán

Eloy Mosqueda Tapia

\section{Resumen}

Perla Shiomara del Carpio Ovando

Se podría pensar que por definición el migrante se desempeña en los nichos laborales menos redituables y de exigua movilidad social. Sin embargo, un grupo nutrido de migrantes en diferentes países económicamente desarrollados han emprendido con éxito negocios de diversa naturaleza y volumen. Es por esto que el espíritu empresarial de los migrantes es un tema que ha merecido una creciente atención en los últimos años. Comparados con otros grupos de inmigrantes en Estados Unidos, los mexicanos muestran bajos índices de actividad empresarial. El objetivo del presente trabajo es hacer, a través de una revisión bibliográfica general y de datos estadísticos oficiales, un análisis preliminar del empresariado migrante mexicano en Estados Unidos, que vale decir en los últimos años ha venido creciendo en importancia.

Palabras clave: empresarios migrantes, migración internacional, inversión migrante.

Abstract

You might think that by definition the migrant labor plays in less profitable niches and meager social mobility. However, a large group of migrants in different economically developed countries have successfully launched businesses of diverse nature and volume. This is why entrepreneurship of migrants is an issue that has received increasing attention in recent years. Compared to other immigrant groups in the United States, Mexicans show low levels of entrepreneurial activity. The aim of this paper is to, through a general 
literature review of official statistical data, a preliminary analysis of mexican migrant entrepreneurship in the United States, that is to say in recent years has been growing in importance

Key words: migrant entrepreneurs, international migration, migrant investment.

\section{INTRODUCCIÓN}

El caudal de descubrimientos teóricos y empíricos actuales enfatiza no únicamente los cambios acaecidos, sino la gran complejidad que ha adoptado el proceso migratorio contemporáneo. Así, tenemos que los esquemas migratorios de larga duración sobreviven con nuevas modalidades, que surgen como consecuencia de los nuevos patrones de acumulación, los vertiginosos cambios económicos, las transformaciones en la ciencia y la tecnología, los novedosos medios de comunicación y transporte, las luchas políticas, los conflictos violentos, las persecuciones religiosas e inter-étnicas, las batallas contra el narcotráfico, los eventos meteorológicos, los cambios climáticos y la degradación del medio ambiente ${ }^{1}$. Logrando con ello complejizar a un más este fenómeno en sus causas y consecuencias tanto en las sociedades de origen como de destino.

Para Castles y Miller (2004) una de las características que definen la etapa posterior a la guerra fría ha sido la importancia creciente de la migración internacional en todas las regiones del mundo. Entre sus características más notorias están el crecimiento de los flujos entre fronteras de diversos tipos, lo cual incluye la inversión, el comercio, los productos culturales, las ideas y las personas; y la proliferación de redes transnacionales con nodos de control en múltiples localidades. Asimismo, enfatizan que mientras los movimientos de personas a través de las fronteras han dado forma a estados y a sociedades desde tiempos inmemoriales, lo que es distinto en años recientes es su alcance global, su carácter central para la política doméstica e internacional, y sus

${ }^{1}$ Aunque las variables de clima y medio ambiente a lo largo de la historia del fenómeno migratorio han sido factores fundamentales en ciertos desplazamientos de población, hay autores que consideran que en la actualidad ha adoptado tintes muy preocupantes. Incluso consideran que el cambio climático ya ha desencadenado importantes movimientos migratorios a consecuencia de fenómenos meteorológicos extremos, la subida del nivel del mar y la rapida degradación del medio ambiente. Hay quienes siguieren estiman que llegaŕn incluso a 1000 millones. De darse esto estaremos en un escenario de escalas nunca vistas (McLenan, 2011; OIM, 2012) enormes consecuencias económicas y sociales.

\section{CONTEXTO DE LA MIGRACIÓN CONTEMPORÁNEA \\ MÉXICO-ESTADOS UNIDOS}

Es innegable la importancia del fenómeno de la migración a nivel mundial. Según cifras de la International Organization for Migration (2012) y del Consejo Nacional de Población (2012) en 1965 se reportaron 65 millones de migrantes internacionales, aumentando significativamente a 105 millones para el año de 1985 y 214 millones (3.1\% de la población mundial) para el año 2010. Si juntáramos a todos los trotamundos en un solo país su población constituiría el $5^{\circ}$ país más poblado del planeta, con un $49 \%$ de mujeres. Existe también un cantidad considerable de desplazados internos en el mundo ( 27.5 millones) en 2010, más de 6 millones que se reportaron en el año 2000; y 15.4 millones de refugiados.

Estados Unidos sigue siendo el país con mayor recepción de inmigrantes. Para el año 2010 este país alcanzó los 310 millones de habitantes de los cuales 42 millones eran inmigrantes, lo que significa que este grupo constituye el $13.8 \%$ del total de la población de esa nación, y el $20 \%$ de los migrantes a nivel mundial, muy por encima de Rusia, segundo lugar, que capta el $5.7 \%$ de los migrantes internacionales (CONAPO, 2010).

En las últimas décadas el patrón migratorio proverbial México-Estados Unidos ha tenido cambios en cuanto a su magnitud, intensidad, modalidades y características, sellando con ello un nuevo ciclo en este fenómeno (Borjas y Katz, 2005). Así lo atestiguan los estudiosos del fenómeno migratorio a Estados Unidos que coinciden en señalar que las características de este nuevo panorama difieren de manera muy notable con las registradas en periodos anteriores. Y las repercusiones en el ámbito sociocultural no son menos significativas, destacando entre ellas la expansión de las organizaciones transnacionales, los cambios en las prácticas comunitarias y las tensiones en los procesos de identidad y socialización de las nuevas generaciones (Ariza y Portes, 2007).

México es el principal país exportador de recursos humanos en el mundo donde $98 \%$ de sus migrantes residen en Estados Unidos. La cantidad de mexicanos en la Unión Americana pasó de 800 mil en 1970, 8.7 millones en 2000 a 11.9 en 2010 (10.62\% de los 112 millones de habitantes en México que reportó el Censo de Población y Vivienda del INEGI 2010), con un flujo 
migratorio menos dinámico en la segunda mitad de ese periodo, observándose en 2008 un flujo negativo en 326 mil personas², pero resultó positivo en 2009 y 2010 (Cervantes, 2011).

$\mathrm{Si}$ a esa cifra le sumamos la población de origen mexicano, su monto aumenta a poco más de 33 millones para 2010, 21.2 millones nacieron en Estados Unidos (11.2 millones de segunda generación y 9.9 millones de tercera generación). Estas cifras ubican a México como el país con mayor inmigración en Estados Unidos por encima de grandes regiones del mundo como Asia, Europa y el resto de América Latina. Actualmente el $4 \%$ de la población total de Estados Unidos son mexicanos y alrededor del $30 \%$ de la población migrante (CONAPO, 2010). Está claro con esto, afirman Borjas y Katz (2005), que los inmigrantes mexicanos en estos últimos años no tiene precedentes históricos, es numérica y proporcionalmente el más grande grupo de inmigrantes a lo largo del siglo XX en Estados Unidos.

Por eso no es de sorprender la importante suma de dinero que fluyó en términos de remesas, que a pesar de la problemática económica muy sentida en los últimos años, siguieron reportando cantidades considerables. En 2010, según datos de la IOM (2012), el monto de remesas se estima en 440 mil millones de dólares a nivel mundial, muy por encima de 132 mmd que circularon en el año 2000. Los principales países beneficiarios para el 2010 fueron India, China, México y Filipinas. Los países ricos son la principal fuente de remesas, y Estados Unidos lidera este renglón ya que registró 48300 millones de dólares en el 2009. Es decir, tomando los datos del BBVA/Research (2012), el crecimiento de las remesas en el mundo entre 1990 y 2010 tuvo un incremento de 6.4 veces, muy por encima del 1.4 veces que experimentó en crecimiento de los migrantes internacionales en el mismo periodo.

Para Cervantes (2011) el ingreso a México por concepto de remesas, aunque modesto en relación al tamaño de la economía mexicana (equivalente en 2010 a $2.1 \%$ del PIB), ha sido significativo en su magnitud absoluta y muy positivo para paliar los niveles de pobreza para millones de familias receptoras. Estos recursos del exterior han permitido que dichas familias tengan mejores niveles de bienestar y acceso al consumo, educación, salud, vivienda, y una parte de ellas, a los negocios familiares. Así tenemos que la evolución del

${ }^{2}$ El Pew Hispanic Center (PHC) establece que al parecer los factores que detuvieron, y retrocedieron, el flujo migratorio de mexicanos a la "gabacho" fueron el debilitamiento del mercado laboral, y muy en especial el de la construcción de viviendas, en Estados Unidos; la contención fronteriza y el mayor peligro, por ende, las tasas de natalidad de México y más amplias condiciones económicas en México. Es posible, según el PHC que la inmigración de mexicanos se reanude cuando se recupere la economía de Estados Unidos. ingreso por remesas familiares ha tenido un crecimiento muy acelerado en los últimos años. En 1999 fueron de 5.9 mmd llegando a un poco más de los 26 mmd en 2007, descendiendo a los 21.2 mmd para el 2010.

Además de la importancia de la migración internacional para México, en las últimas décadas asistimos a una presencia cada vez más grande de los empresarios migrantes mexicanos que han invertido en territorio estadounidense. El objetivo del presente trabajo es hacer un primer acercamiento, a través de una revisión bibliográfica sobre la literatura especializada y los datos estadísticos, del empresariado mexicano en Estados Unidos. El alcance por lo tanto es modesto.

En futuras investigaciones pretendemos ahondar en estudios de caso del fenómeno de la inversión migrante. Esta investigación tiene tres aparatados generales: una breve explicación de las teorías en torno al empresariado migrante; una revisión bibliográfica de la literatura especializada en los migrantes inversores en los países de destino y, finalmente, un análisis del empresariado migrante mexicano en Estados Unidos tomando en cuenta datos estadísticos y teórico conceptuales, a través de coordenadas sociodemográficas como escolaridad, estatus migratorio, lugares de destino o elementos más cualitativos como adaptación a la sociedad estadounidense y lazos transnacionales.

\section{TEORÍAS SOBRE EL EMPRESARIADO MIGRANTE}

La literatura académica ha utilizado diversas teorías para explicar al empresariado migrante. Entre estas se encuentran la Teoría Cultural, la Teoría de la Desventaja (Disadvantage) y la Teoría de la Incrustación Mixta (Mixed Embeddedness). Según la Teoría cultural (Azmat, 2010) las características culturales como creencias religiosas, lazos familiares, ahorros, trabajo étnico, y el cumplimiento con los valores sociales sirven como recursos étnicos, el cual parcialmente explica la orientación de los inmigrantes hacia la actividad empresarial. Las variaciones entre grupos de inmigrantes en sus estrategias de negocios, resultados económicos y la intensión de autoemplearse puede ser explicado por las diferencias culturales entre los países. Esta teoría centra sus baterías analíticas en los aspectos culturales con una falta de atención en el ambiente y contexto socioeconómico en el cual operan los negocios (González, Bretones, Zarco y Rodríguez, 2011). Al respecto, Ibrahim y Galt (2011) consideran que examinar el empresariado étnico solamente en términos culturales es limitado. Este ignora el hecho de que la cultura esta incrustada en un contexto 
histórico. O como bien dijera Fairchild (2010), las condiciones históricas son importantes en la evolución de la actividad empresarial dentro de una región.

La Teoría de la incrustación mixta se concentra en la incrustación de los empresarios en el ambiente socio-económico y político-institucional del país de destino y cómo esas fuerzas perfilan las oportunidades de los empresarios. Los afines a esta teoría destacan además del mercado, políticas estatales y configuración espacial de los empresarios migrantes, el sector, localidad, mercado de trabajo y el apoyo institucional. Y la Teoría de la desventaja plantea que las actividades del empresariado migrante son resultado de un contexto de desventaja. Algunos autores observan que una porción importante de trabajadores inmigrantes están sujetos a un mercado laboral en desventaja debido a su acento y el no reconocimiento de credenciales en el extranjero. Otros ponen la atención en los frecuentes fracasos empresariales y escasa rentabilidad de gestión creada por los empresarios migrantes. Esos fracasos pueden ser mayormente atribuidos a una variedad de condiciones del mercado que a los empresarios migrantes se les presenta en los países desarrollados y que no estaban consolidadas en países en vías de desarrollo, como la regulación de negocios y mercado laboral, requisitos de aprendizaje, normas sanitarias, salarios mínimos que impiden que se desarrollen los negocios étnicos (Aznat, 2010).

Una visión más integral y holística, para entender la formación de los negocios de los empresarios inmigrantes, proponen Ibrahim y Galt (2011) donde se contemplen factores tanto de orden económico como cultural, que puntualicen la elección racional, la dependencia de la senda y la evolución dinámica de las acciones y el comportamiento. Ilham, Sahin y Calingir (2011) en este mismo sentido proponen un modelo para entender al empresariado migrante. Los autores sugieren analizarlo en el contexto del país de origen y destino en cuatro grandes coordenadas. Primero, antecedentes: factores pull-push, experiencia empresarial, redes sociales, política, género, capital humano, factores demográficos, historia; segundo, contexto ambiental: contexto socioeconómico e institucional, raíces culturales, factores financieros, mercado laboral y contexto geográfico; tercero, características del espacio empresarial internacional: escala, alcance industrial y factores estructurales; y cuarto, resultados: a nivel individual (oportunidades de empleo y beneficios económicos), nivel organizacional (pequeñas empresas), y a nivel país (inmigrantes empresarios trasnacionales, mercado laboral y enclave étnico).

\section{EL EMPRESARIADO MIGRANTE COMO OBJETO DE ESTUDIO}

Para los estudiosos del fenómeno migratorio bien es conocido encontrar en Ravenstein el pionero de esta temática a finales del siglo XIX. Desde entonces hasta la actualidad, la bibliografía sobre migración es abrumadora. A ella han asistido con profusión y creatividad demógrafos, sociólogos, historiadores, economistas, abogados, antropólogos, políticos e individuos sin credenciales académicas, pero que con gran deleite han vertido sus vivencias de trotamundos. En las bases de datos, bibliotecas, hemerotecas exploramos atónitos narraciones pretéritas, transformaciones sociales, reacomodos en los patrones de consumo y familiares en sus roles, el afloje de tuercas emocionales, las victimas del desierto inexpugnable, las costumbres y tradiciones trastocadas, las variables cuantitativas de los que se van y se quedan, los amores fugados, impactos de las remesas en el desarrollo, de los movimientos internos, el desequilibrio ecológico, la inseguridad. El tema migratorio toca todas las disciplinas y saberes sociales.

Se podría pensar que por definición el migrante se desempeña en los nichos laborales menos redituables y de exigua movilidad social. Sin embargo, un grupo nutrido de migrantes en diferentes países económicamente desarrollados han emprendido con éxito negocios de diversa naturaleza y volumen. Es por esto que el espíritu empresarial de los inmigrantes es un tema que ha merecido una creciente atención en los últimos años (Tamer, Zamantili y Hellstern, 2011). Veamos cómo lo han abordado.

En la década de 1980, Chung y Moo (1985) observan que una cantidad considerable de coreanos participaban activamente en pequeños negocios de autoempleo en Chicago, Estados Unidos. En esta investigación los autores descubren que los empresarios coreanos dependen en gran medida de sus recursos étnicos para la preparación y operación de sus negocios. Pero enfáticos afirman que, por un lado, la utilización de tales recursos les brinda ventajas competitivas, el mismo mecanismo plantea serios problemas, como la competencia de los negocios intra-étnicos y una posición precaria como una intermediaria minoría.

En los noventa las investigaciones sobre los empresarios migrantes se incrementan. En un trabajo de corte histórico Bovenkerk y Ruland (1992) estudiaron al migrante empresario artesanal italiano en Holanda. A través de la historia oral y el meticuloso trabajo de archivo en ambos países y con entrevistas de los descendientes de migrantes retornados en los pueblos de 
origen, así como con los relatos de los historiadores locales, reconstruyen este devenir histórico que hunde sus raíces en la baja edad media y que continúa bien entrado el siglo XX.

Por su parte Yuengert (1995) en una investigación de corte teórica pone en duda la hipótesis del enclave étnico. En este estudio descubre que los inmigrantes de países con más grandes sectores de autoempleo tienen más altas tasas de autoempleo. Los migrantes a Estados Unidos se concentran más en los estados con altos impuestos y encuentran más grandes oportunidades para las reducciones de impuestos como empresarios que como trabajadores asalariados.

Portes y Zhou (1996) en un muy interesante trabajo que trasciende los estrechos márgenes economicistas afirman que la presencia de un grupo de exitosos empresarios inmigrantes puede tener efectos sociales y económicos en sus comunidades que van mucho más allá del éxito puramente individual. A finales de esa década varios investigadores centraron su atención en la actividad empresarial de varios grupos de inmigrantes en Gran Bretaña. Por un lado, Basu (1998) al analizar los pequeños negocios de los asiáticos en ese país encontró que no hay ninguna evidencia de que estos grupos étnicos fueran empujados al trabajo autónomo para evitar el desempleo. Su éxito económico como empresarios parecía estar positivamente asociado con la participación del capital personal invertido en el comienzo y cualidades educativas. Por su parte, Borooah y Hart (1999) estudiaron las diferencias en el auto-empleo entre los hombres hindúes y negros caribeños en Gran Bretaña. Ellos encontraron que los atributos sociales relacionados a la formación familiar son por lo menos tan importantes como las características individuales.

Con la nueva centuria la proliferación de investigaciones de esta naturaleza es evidente. Hay trabajos teóricos de manufactura muy sobresaliente. Es el caso de lo publicado por Portes, Guarnizo y Hallen (2002) que desde un enfoque del transnacionalismo proponen que en lugar de concentrarse en asuntos tradicionales acerca de los orígenes de los inmigrantes y su adaptación a las sociedades receptoras, esta perspectiva se enfila en la continuidad de las relaciones entre inmigrantes y sus lugares de origen y cómo ese ir y venir construye campos sociales complejos que se sitúan en las fronteras nacionales. Para estos investigadores la empresa económica transnacional es uno de los más grandes potenciales que afectan la movilidad socioeconómica de los inmigrantes y su influencia en las comunidades de origen. Arrastrado el enfoque del transnacionalismo Riddle, Hrivnak y Nielsen (2010) utilizan el concepto de diáspora empresarial que vendrían ser los migrantes y sus descendientes quienes establecen actividades que abarcan los entornos empresariales nacionales de sus países de origen y de destino. Este trabajo es un estudio de caso de IntEnt, una incubadora que brinda servicios exclusivamente a la diáspora empresarial transnacional. Los autores exploran cómo los negocios de incubadoras contribuyen al desarrollo económico de los mercados emergentes.

Con el mismo enfoque del transnacionalismo aunado con una visión de la Teoría institucional Riddle y Brinkerhoff (2011) analizan el caso de la empresa Thamel.com. El objetivo de esta investigación es observar cómo las instituciones y las reglas de juego son fundamentales para la creación y desempeño de los negocios de las diásporas empresariales transnacionales. Y el asunto para ellos es que las debilidades institucionales en muchos países en desarrollo impiden la inversión extranjera y constriñen el espíritu empresarial en esas economías, empujando a los individuos a dejar su país de origen en busca de oportunidades en el extranjero.

Otras investigaciones que, por su gran alcance de corte teórico, podemos mencionar son las realizadas por Ilhan-Nas, Sahin y Cilingir (2011). Usando un análisis contextual, este artículo revisa la literatura sobre el espíritu empresarial internacional en términos de temas de investigación, métodos de investigación, análisis aplicados, resultados y contexto ambiental. Este exhaustivo estudio revisa artículos entre 1936 y 2008. Por su parte, Ibrahim y Galt (2011) se preguntan por qué hay disparidades en el monto y espíritu empresarial entre los diferentes grupos étnicos. Apoyándose en los economistas institucionales, en el modelo del path dependency (dependencia de la trayectoria) y el comportamiento evolutivo intentan explicar la presencia desproporcionada del empresariado étnico en los países de destino.

En diferentes geografías se ha intentado develar las características de la actividad empresarial de los inmigrantes. A través de la metodología cualitativa. Pio (2007) por eso explora las experiencias de vida de las empresarias hindúes en Nueva Zelanda en el contexto del espíritu empresarial étnico. Le interesa observar variables como: discriminación, baja autoestima, redes étnicas y la carencia de acceso y conocimiento de los recursos gubernamentales para entender el proceso de ingreso al mercado laboral, desempleo, creación de empresas.

Se requieren de ciertas cualidades de los migrantes tales como: autoconfianza, competencia social, alto nivel educativo y motivación para establecer su propio negocio en los países huésped. Tal es la afirmación de Dai, 
Yuan y Teo (2011) al analizar la actividad empresarial de los migrantes chinos en Australia y en países de occidente que se dedican a los negocios de la venta directa (network marketing).

Europa en los últimos años ha sido escenario de una creciente actividad empresarial migrante. Es el caso de Holanda donde Nijkamp, Sahin y BaycanLevent (2009) establecen que los empresarios migrantes forman una parte significativa de las pequeñas y medianas empresas en las ciudades holandesas y pueden, por lo tanto, ser importantes vehículos para la vitalidad urbana. Para el caso de Alemania Constant, Shachmurove y Zimmermann (2007) analizan al empresariado turco en este país. Dicho estudio apunta a que la educación no es decisiva a la hora de determinar la elección del empleo independiente por delante del asalariado ni a la hora de explicar los ingresos. Por su parte, resulta muy sugerente el trabajo realizado por Vinogradov y Kolvereid (2010) cuando analizan a los emprendedores migrantes en Noruega.

Según estos investigadores el nivel de autoempleo varía significativamente entre los inmigrantes de diferentes países de origen. El objetivo de esta investigación es examinar la relación entre inteligencia del país de origen y las tasas de empleo por cuenta propia entre inmigrantes de primera generación en ese país escandinavo. En este estudio los afortunados resultaron ser los asiáticos que han mantenido durante más largos periodos de tiempo, a nivel individual y de grupo, un nivel de inteligencia nacional más alto.

Resulta muy interesante la investigación emprendida por González, Bretones, Zarco y Rodríguez (2011) que a través del método cualitativo basado en entrevistas semiestructradas examinan -desde una perspectiva de género psicosocial- el comportamiento empresarial de 52 mujeres inmigrantes viviendo en España. En sus conclusiones afirman que las tasas y el espíritu empresarial entre hombres y mujeres son asimétricos, ya que hay rasgos de la personalidad empresarial, valores personales y motivaciones muy disimiles.

En el continente americano la actividad empresarial y su análisis ha estado muy concentrada en Canadá, y muy en especial en Estados Unidos. Para el caso de Canadá, Li (2001) observa que los inmigrantes presentan ya en esos años más altas tasas de autoempleo que los nativos canadienses. Estados Unidos, como es muy conocido, es el país de la inmigración por antonomasia, y en él la actividad empresarial de los migrantes y su análisis académico en los últimos años ha sido muy dinámico. Tenemos el caso de los empresarios inmigrantes chinos y coreanos que jugaron un rol muy importante en la inversión, promoción de la inmigración co-étnica a Los Ángeles, la creación y preparación de nuevos vecindarios residenciales para los nuevos inmigrantes (Light, 2002).

Bogan y Darity (2008) analizan y comparan la evolución del espíritu empresarial entre afroamericano e inmigrantes. Este artículo examina cómo las fuerzas sociales, económicas y políticas han influido negativamente para el desarrollo empresarial de la población de color comparado con varios grupos de inmigrantes. Usando datos censales de los años noventa, los investigadores encontraron evidencia empírica que sustenta la aseveración que muchos inmigrantes tienen recursos (no disponibles a los nativos no blancos) que facilitan el espíritu empresarial.

Fairchild (2010) se pregunta ¿Cómo la experiencia de vida en un enclave étnico durante los años formativos influye para convertirse en empresarios? En este estudio el autor examina la influencia intergeneracional y de los vecinos co-étnicos en la decisión de los migrantes en convertirse en empresarios.

Hay investigaciones con un enfoque procedimental y cultural muy evidente. Este es el caso de Azmati (2010), quien afirma que la globalización y los avances tecnológicos han difuminado las barreras geográficas trayendo como resultado el incremento del empresariado migrante. Sin embargo, estos empresarios muestran desafíos como resultado de diferentes valores, políticas, ambiente institucional, cultura y percepción de la responsabilidad social en el país de destino. En este artículo Azmati se centra sobre uno de estos desafíos, explora si las percepciones de la responsabilidad social de los empresarios migrantes procedentes de los países menos desarrollados se ven influidos por los factores contextuales del país de origen tales como: cultura, ambiente institucional y nivel de desarrollo socioeconómico.

El autor es tajante en afirmar que los empresarios migrantes procedentes de países poco desarrollados del sur de Asia y África tienen más dificultades para tener éxito en los países de destino, ya que están acostumbrados a operar en un ambiente donde hay pocos estándares de responsabilidad social, baja presión pública y donde las regulaciones y códigos de conducta están limitados.

Kesler y Hout (2010), por su parte, utilizan de una manera muy interesante el concepto de movilidad social ascendente para analizar ciertos grupos de inmigrantes empresarios en Estados Unidos. Para estos autores convertirse en empresario requiere recursos financieros, humanos y capital social, y la disponibilidad de esos recursos varia de un grupo a otro. Algunos grupos, por lo tanto, tienen más altas tasas de autoempleo que otros.

Los fuertes lazos involucrados en las comunidades étnicas de las poblaciones migrantes han sido considerados los activos clave para los empresarios 
migrantes. Sin embargo, según Yang, Colarelli, Han y Page (2011) poca investigación se ha realizado sobre cómo el parentesco biológico y las teorías biológicas del altruismo influyen en el comportamiento del empresariado étnico. En esta investigación los autores aplican la teoría evolucionista neodarwiniana del parentesco para examinar funciones adaptativas de parentesco y altruismo étnico en la creación de empresas y las prácticas de contratación de los empresarios migrantes coreanos en Estados Unidos.

Por su parte, Chand y Ghorbani (2011) hacen un estudio comparativo entre comunidades chinas e indias en Estados Unidos. Este trabajo usa una combinación de un enfoque de cultura nacional y teoría de capital social para explicar la formación y gerencia de proyectos empresariales entre estos grupos étnicos.

La participación del empresariado migrante latino en Estados Unidos en los últimos años ha crecido. A esta conclusión llegan Robles y CorderoGuzmán (2007) quienes establecen que aunque se ha puesto mucha atención al crecimiento de la población latina y su contribución al mercado laboral en Estados Unidos, poca atención se ha prestado al crecimiento del espíritu empresarial y negocios latinos. Por lo tanto, hay un gran vacío en cuanto a su conocimiento. Para el caso concreto de los empresarios migrantes mexicanos en Estados Unidos varios trabajos dan cuenta de su actividad.

Raijman (2001) profundiza sobre las intensiones de inversión de los migrantes mexicanos en Chicago, Estados Unidos. Esta investigación, utilizando variables tales como: disposición al riesgo, familiares empresarios y recursos económicos en el hogar, se centra en los inmigrantes mexicanos que están pensando en iniciar un negocio, pero no lo hacen por diversas razones. En su investigación, Fairlie y Woodruff (2007) exploran posibles explicaciones de los porcentajes más bajos de empresarios entre los inmigrantes mexicanos en Estados Unidos. Y pueden ser muy escasos debido a las características que los migrantes en Estados Unidos tienen a diferencia de los mexicanos que se mantienen en México. Los migrantes mexicanos.

Por su parte, Georgarakos y Tatsiramos (2009) afirman que muchos estudios han explorado las determinantes para entrar en el espíritu empresarial y las diferencias y montos del autoempleo a través de los grupos raciales y étnicos. Sin embargo, muy poco se conoce acerca de la sobrevivencia del espíritu empresarial de los inmigrantes en Estados Unidos y sus descendientes. Los autores encontraron baja probabilidad de sobrevivencia del espíritu empresarial para los mexicanos y otros inmigrantes latinos, ya que su actividad como empresarios brotó como una alternativa al desempleo, sugiriendo que su emprendimiento empresarial representó para ellos un paso intermedio desde el no empleo al empleo pagado. Por ello, los hispanos y afroamericanos exhiben muy bajas tasas de negocios comparado con los blancos y asiáticos.

Shinnar, et a./(2009) tratan de entender las diferencias entre la experiencia empresarial de los inmigrantes mexicanos y los mexicanos nacidos en Estados Unidos. Los resultados sugieren que aun dentro de un particular grupo minoritario, hay significativas diferencias entre empresarios inmigrantes $y$ nacidos en Estados Unidos. Por ejemplo, los mexicanos nacidos en la Unión Americana están más motivados por individualistas beneficios financieros de ser empresarios, mientras que los empresarios inmigrantes mexicanos están más motivados por servir a la sociedad y a su comunidad co-étnica. En su investigación Fairlie y Woodruff (2007) exploran posibles explicaciones de los más bajos porcentajes de empresarios entre los inmigrantes mexicanos en Estados Unidos. Pueden ser muy bajos debido a las características que los migrantes en la Unión Americana tienen a diferencia de los mexicanos que se mantienen en México. Los migrantes mexicanos en aquel país difieren en edad y educación que la población residente en México. Así, edad y educación son importantes determinantes en los porcentajes de autoempleo en Estados Unidos.

\section{EMPRESARIOS MEXICANOS EN ESTADOS UNIDOS:}

\section{UN ACERCAMIENTO PRELIMINAR}

\section{ESCOLARIDAD}

Basándose en cifras oficiales de Estados Unidos, Albo y Ordaz (2011) anotan que en el año de 2009 radicaban en la Unión Americana poco más de 20 mil migrantes mexicanos con estudios de doctorado. El 46\% (9 383) de ellos migró a ese país en las dos últimas décadas; el 34\% (6 832) en las décadas de los setenta y ochenta y el otro 20\% (4 002) antes de 1970.

Para inicios del 2009 había poco más de 80 mil personas radicadas en la República Mexicana con estudios de doctorado, de los cuales 73 mil nacieron en suelo mexicano. Por lo que tenemos que alrededor del $20 \%$ de las personas nacidas en México con títulos doctorales viven en Estados Unidos. Es decir, es casi el doble en términos relativos del total de migrantes mexicanos en el vecino país del norte, que es de $11 \%$. Datos también muy relevantes, si los 
comparamos con el total de miembros del Sistema Nacional de Investigadores de México que oscila entre los 16 mil y 19 mil integrantes. Consideran también estos autores que la probabilidad de que un mexicano con doctorado migre a Estados Unidos es cuatro veces mayor a la de un mexicano con primaria y tres veces mayor con secundaria.

En los últimos 20 años el promedio de escolaridad de los migrantes mexicanos se ha incrementado. Se han observado mayores flujos migratorios en las personas con estudios de nivel medio superior, y visiblemente ha habido un aumento del grupo con nivel técnico, superior y posgrado. De ello se puede inferir que la migración mexicana a Estados Unidos ha incrementado su cualificación laboral. El número de migrantes con 10-12 grados de escolaridad aumentó tres veces entre 1994-2009 y el nivel técnico, profesional y posgrado lo hicieron al doble cada uno. Así tenemos que el promedio de escolaridad de los migrantes mayores de 15 años es de 9 años, mientras que el promedio de escolaridad en México es de 8 años. De los casi 12 millones de migrantes mexicanos en Estados Unidos el 37\% tiene el nivel preparatoria (Albo y Ordaz, 2011).

\section{NATURALIZADOS ESTADOUNIDENSES}

Según la Homeland Security (2011) en el año de 2011 el número total de personas naturalizadas fue de 694 193. Los nuevos ciudadanos fueron en su mayoría de origen mexicano (94 783), seguido de la India, Filipinas, China y Colombia. Para hacerse ciudadano estadounidense se requiere ser mayor de 18 años, haber tenido el estatus de residente permanente, haber residido en el país cinco años de manera continua por lo menos. Adicional a ello, se demanda hablar, leer y escribir en inglés, conocimiento de la historia y del gobierno de Estados Unidos y un "buen carácter moral".

El promedio anual de personas que se naturalizan se incrementó al menos durante las décadas de 1950 y 1960 a 120 mil, 210 mil durante el decenio de 1980, 500 mil en la década de1990 y 680 entre el año 2000 y 2009. Hasta la década de 1970 la mayoría de las personas naturalizadas eran de países europeos.

La escolaridad y el estatus migratorio pueden ser variables para potencializar la actividad empresarial en Estados Unidos de los migrantes mexicanos. Los migrantes altamente calificados poseen un fuerte capital humano capaz de habilitar un activismo empresarial, y con el estatus de ciudadanía se adquieren derechos y una mayor posibilidad de aprovechar los reductos institucionales para tramitar la apertura de nuevas empresas. Pero el espíritu empresarial va más allá de elementos procedimentales y habilidades altamente especializadas.

\section{LUGARES DE DESTINO EN ESTADOS UNIDOS}

De acuerdo con el Pew Hispanic Center en el 2010 se reportó una población total de origen hispano en Estados Unidos de 50, 730, 000 personas. De las cuales casi 33 millones son de origen mexicano (64.9\%), le siguen en importancia Puerto Rico con 4.6 millones, Cuba con 1.8 millones y El Salvador con 1.8 millones.

De la población de origen mexicano el 36\% nació en México, el 26\% cuenta con High School, el 9\% con nivel bachillerato o más, el 64\% son competentes en el idioma inglés; $73 \%$ tiene la ciudadanía estadounidense; el ingreso medio por hogar de 38700 dólares anuales; $27 \%$ viven en la pobreza, porcentaje más elevado comparado al promedio general en Estados Unidos que fue de 15\%; el $34 \%$ no tiene seguro de salud y el $50 \%$ son propietarios de sus casas. Y las ciudades donde están más concentrados son Los Ángeles, California, con 3.5 millones; Harris Co. Texas, con 1.2 millones; Maricopa Co. Arizona, con 975622. De las 10 ciudades estadounidenses que albergan más habitantes de origen mexicano cinco están en California y tres en Texas.

Según las estadísticas del Pew Hispanic Center, la mayoría de los hispanos viven en nueve estados de Estados Unidos: Arizona, California, Colorado, Florida, Illinois, Nuevo México, Nueva Jersey, Nueva York y Texas. En el año 2010 el $76 \%$ de los latinos radican es esos estados, comparado con el 81\% en 2000 y $86 \%$ en 1990. En el año 2000 la mitad vivía en California y Texas, ya para 2010 fue de $46.5 \%$. Hay ocho estados que superan el millón de hispanos, California es el que concentra la mayor cantidad con 14 millones.

Al igual que los hispanos, en los últimos años se observa una mayor diversificación de los lugares de destino de los migrantes mexicanos en Estados Unidos (Díaz, 2008). Si en los años de la década de 1930 el destino predilecto de los mexicanos fue Texas, ya para 1960 California lo desplazó. La región del Sudoeste integrada por Arizona, California, Nuevo México y Texas en 1990 albergaba al 83.2\% de los inmigrantes mexicanos, y en el 2010 cayó al 66\%. La presencia de los mexicanos en la región de los Grandes Lagos, principalmente en Illinois, data de inicios del siglo XX (Taylor, 1930), y es precisamente este estado quien ocupa el tercer lugar, tan solo superado por California y Texas. Chicago concentra alrededor del $90 \%$ de los mexicanos en Illinois y es la segunda ciudad más poblada de mexicanos, después de Los Ángeles, California 
(CONAPO, 2010)

En base a las cifras manejadas por el Current Population Surveyentre los años 2010-2011 un 85.9\% de los inmigrantes mexicanos en Estados Unidos se mantuvo en el lugar de residencia y el $14.1 \%$ si se movió $(12.5 \%$ al mismo estado; $1.1 \%$ a otro estado; $0.4 \%$ a otro país). En 2011 la distribución regional de los mexicanos en Estados Unidos estuvo concentrada en el Oeste (51.4\%), Sur (35.7), Medio Oeste (10.1\%) y Noreste (2.8\%).

Contrario a lo que muchos pensarían, México es uno de los más emprendedores países en el mundo. Por los índices mostrados en cuanto a trabajo por cuenta propia en el año 2000 la OCDE clasificó a México en la parte superior de la lista de los 28 países miembros. A través de fuentes internacionales y de los datos estadísticos oficiales en México, Fairlie y Woodruff (2007) indican que aproximadamente un cuarto de la fuerza de trabajo en México es propietario autónomo de un negocio a inicios de la década pasada. En Estados Unidos, sin embargo, los índices de empresarios migrantes mexicanos son bajos. Solamente el $6 \%$ lo es (porcentaje por debajo del promedio nacional del $11 \%$ ). Nada comparado con el $31 \%$ de los griegos, $27 \%$ de los coreanos, $21 \%$ de los italianos y el $13 \%$ de los brasileños.

Hay autores que dan una fuerte preeminencia a la genética empresarial, la experiencia abrevada del espíritu empresarial familiar. En este sentido, Fairlie y Robb (2007) examinan en su trabajo por qué las empresas de propiedad de afroamericanos están más atrasadas que las empresas de propietarios blancos, en ventas, beneficios, empleo y sobrevivencia. Los autores consideran que las empresas de afroamericanos son mucho menos propensas que la de los blancos de haber tenido una familia propietaria de autoempleo antes de comenzar su negocio y menos probabilidades de haber trabajado en la empresa familiar. Los autores descubrieron que la carencia de previa experiencia en una empresa familiar entre los propietarios negros, quizá al limitar su adquisición de capital humano general y específico de los negocios, afecte negativamente sobre los resultados de los propietarios afro.

Fairlie y Woodruff (2007) afirman que los individuos quienes han tenido un padre empresario tienen aproximadamente el doble o triple de posibilidad de ser empresarios que quienes no tuvieron un padre con experiencia empresarial. Igual aseveran Fairlie y Robb (2007), quienes señalan que la probabilidad de tener una empresa es sustancialmente más alta entre los hijos de autoempleadores. Si nos atenemos a los datos anteriores, las posibilidades de los migrantes mexicanos de convertirse en empresarios vía influencia de sus padres, es alta. Sin embargo, trasladados a otros escenarios los migrantes mexicanos no continúan con la tradición empresarial en Estados Unidos.

Otro elemento a considerar es el aspecto institucional. Hay investigadores, como Azmat (2010), quienes consideran que los empresarios migrantes que proceden de países subdesarrollados se enfrentan a desafíos en el país receptor, tienen que someterse a legislaciones y reglas estrictas que en sus países de origen no están acostumbrados a lidiar. Los factores culturales del país de origen, que incluyen valores, actitudes, reglas informales, creencias religiosas y reglas de conducta, son probables que tengan una fuerte influencia en la configuración de las percepciones de la responsabilidad étnica y social en su país. Cuando los empresarios migrantes comienzan sus negocios en el país de destino, ellos se enfrentan a valores contrastantes, creencias, actitudes y prácticas empresariales debido a diferentes valores socio-culturales.

Como todos los países tradicionales de recepción, Estados Unidos, entre otros, la política de inmigración desde hace mucho tiempo se entrelaza estrechamente con la política de población. Como los niveles de fertilidad han caído debajo de los umbrales críticos en recientes décadas, la inmigración se ha convertido en el mejor garante, no solo del crecimiento demográfico, sino además del reemplazo de la población y de la fuerza laboral. En Canadá, por ejemplo, cerca del $70 \%$ del crecimiento de la fuerza laboral está atribuida a la inmigración. Un mayor vínculo directo entre política económica y política de inmigración aparece en el intento de los Estados por reclutar trabajadores técnicos altamente calificados en varios campos del conocimiento científico y tecnológico. Y no solo atraen a lo más selecto del ámbito académico, sino también introducen programas para atraer empresarios con un historial probado e importante capital económico (Ley, 2003).

Según el Kauffman Index en 2009 el número de personas que reportaron entrar a una actividad empresarial en Estados Unidos alcanzó su punto más alto de los últimos 14 años. En ese año, 0.34\% de la población adulta de 20 a 64 años (340 de cada 100 mil adultos) crearon un nuevo empleo cada mes, representando aproximadamente 558 mil nuevos negocios por mes. La actividad empresarial de 2009 representó un incremento respecto a la tasa de 2008 de $0.32 \%$ y representa el nivel más alto de los últimos 15 años. El porcentaje de la actividad empresarial entre los afroamericanos se incrementó de $0.22 \%$ en 2008 a $0.27 \%$ en 2009. Por su parte, el porcentaje de la actividad empresarial de los latinos decreció de $0.48 \%$ en 2008 a $0.46 \%$ en 2009 , y entre los asiáticos también manifestó un descenso que pasó de $0.35 \%$ en 2008 a 
$0.31 \%$ en 2009 . Y de los blancos no latinos el incremento fue de $0.31 \%$ a $0.33 \%$ en esos mismos años.

Son interesantes los datos que se manejan en el Kauffman Index que establecen que el porcentaje de actividad empresarial de los inmigrantes, aunque declinó en esos años de $0.53 \%$ en 2008 a $0.51 \%$ en 2009 , se mantuvo significativamente más alto que el porcentaje de los nativos cuya tasa fue de $0.30 \%$. Los individuos con educación universitaria se incrementó de $0.31 \%$ a $0.34 \%$, y los de high schoo/(escuela secundaria) aumentó de $0.35 \%$ a $0.38 \%$. La industria de la construcción tuvo el más alto porcentaje de actividad empresarial de todos los grupos industriales más importantes en 2009 con 1.55\%, seguida de la industria de los servicios con $0.42 \%$.

$Y$ el porcentaje de actividad empresarial declinó significativamente en el Oeste que pasó de $0.42 \%$ en 2008 a $0.38 \%$ en 2009 , aunque se incrementó en el Medio Oeste y el Sur, el Oeste mantiene el promedio más alto. Es interesante también observar que los estados que mantienen las tasas más altas de actividad empresarial por cada 100 mil adultos fueron Oklahoma (470), Montana (470), Arizona (460) y Texas (450). Y los estados con menos actividad empresarial fueron Mississippi (170), Nebraska (200), Pennsylvania (200) y Alabama (210). Y en relación a las más grandes zonas metropolitanas en Estados Unidos las tasas más altas de actividad empresarial en 2009 fueron en: Houston, Texas $(0.63 \%)$, Miami $(0.61 \%)$, Phoenix $(0.51 \%)$, Atlanta $(0.51 \%)$, San Francisco $(0.48 \%)$ y Los Ángeles $(0.47 \%)$; y las que reportaron el porcentaje más bajo fue Seattle $(0.16 \%)$ y Philadelphia $(0.18 \%)$.

Según Azmat (2010) se ha descubierto que los negocios de empresarios migrantes son generalmente actividades de pequeña escala, y muestran muchas de las características, problemas y necesidades de apoyo de las micro-empresas. La propiedad de pequeños negocios ha sido una estrategia importante para la adaptación de los inmigrantes a las sociedades desarrolladas.

El capital humano, tales como: las habilidades, educación y experiencia, mercado de capital, el cual incluye medios materiales tangibles relacionados con los antecedentes de clase; y el capital social que son factores intangibles que fomentan la solidaridad de grupo, confianza y obligaciones reciprocas, se combinan para facilitar el espíritu empresarial migrante. Las condiciones históricas son factores importantes a considerar, ya que esto abre la posibilidad de que, bajo ciertas circunstancias favorables, las actividades empresariales tomen cuerpo, así también como contexto político incide en las tasas, crecimiento y expansión de las empresas migrantes. Tanto a nivel macro como micro la política de inmigración, establecimiento y la política fiscal impactan en la formación y sobrevivencia del espíritu empresarial migrante (Ilham, Sahin y Cilingir, 2011).

Las empresas de los inmigrantes tienen una mayor participación en los pequeños negocios en los países desarrollados como Alemania, Inglaterra y Estados Unidos (Ilham, Sahin y Cilingir, 2011). Yang, Colarelli, Han y Page (2011) afirman al respecto que la propiedad de pequeños negocios es frecuentemente considerado un importante vehículo para el ascenso económico y movilidad social para los inmigrantes debido a sus limitadas oportunidades laborales, barreras lingüísticas y discriminación en el país de destino.

El capital es, indudablemente, uno de los más cruciales recursos para la formación de los negocios (Kwang y Won, 1985). Mientras que los empresarios nativos usualmente piden prestado su capital inicial de los bancos, los migrantes empresarios tienen menor posibilidad de recibir fondos del banco que el empresario nativo y, frecuentemente piden prestado capital a la familia o de otros miembros del grupo. Los empresarios migrantes son menos inclinados a reunirse con redes formales nativas, grupos minoristas, asociaciones comerciales, organizaciones de franquicia, etc. Se observa una concentración geográfica, especialmente en las más grandes ciudades, porque los migrantes empiezan sus negocios en lugares donde ya existe una gran población residente de gente con los mismos antecedentes migrantes (Nijkamp, Sahin y Tuzin, 2009)

\section{INTEGRACIÓN A LA SOCIEDAD ESTADOUNIDENSE: ALGUNOS RETOS}

Hay varios factores a considerar para entender la dificultad de los migrantes mexicanos para integrarse a la sociedad estadounidense. Los análisis realizados por la CONAPO (2012) dan cuenta de ello. Según esta institución, a pesar de ser un fenómeno centenario, masivo y continuo, los inmigrantes mexicanos enfrentan múltiples obstáculos para integrase a dicha sociedad comparados con otros grupos migrantes. Esto se refleja en el bajo promedio educativo, escaso manejo del idioma inglés, bajos índices de naturalización, elevada concentración en empleos mal remunerados y a los altos niveles de pobreza de los hogares mexicanos en ese país. Resultados muy escuetos, si los comparamos con los promedios de la población nativa y de otros grupos de inmigrantes. 
Hay investigadores (Fairlie y Woodruff, 2007) que afirman que los inmigrantes son, generalmente, quienes tienen mayor probabilidad de ser empresarios, en comparación a los nativos estadounidenses. Muchos estados y el gobierno federal están promoviendo el autoempleo como una ruta para que los familiares dejen la pobreza y el desempleo. El éxito económico de ciertos grupos de inmigrantes en Estados Unidos como los chinos, japoneses, judíos, italianos, griegos y coreanos radica, en parte, al hecho de que poseen pequeñas empresas.

Existen además evidencias que indican más movilidad ascendente en los trabajadores autónomos de bajos ingresos que los trabajadores asalariados de bajos ingresos, y los empresarios experimentan más rápido crecimiento de ganancias en promedio que los asalariados. Otro argumento para promover el espíritu empresarial entre los inmigrantes es que es un importante vehículo para el crecimiento económico y para la regeneración de las economías (Assudani, 2009). Además, generan empleos en sectores con alto crecimiento potencial.

Los empresarios latinos, eligen como trabajadores, generalmente, a personas también latinas. Los empresarios estadounidenses, por su parte, tienden a contratar a personal de origen diverso.

Visto de ese modo, los empresarios migrantes crean oportunidades de empleo, desarrollo de capital social, una mejor integración dentro de la sociedad, un incremento en el rango de bienes y servicios disponibles, la expansión de alternativas al consumidor y el crecimiento en ciertos sectores (Azmat, 2010). Un ejemplo contemporáneo de empresariado migrante entre las comunidades hindú y china es el Silicon Valley. Los hindúes fundaron el $15 \%$ de todas las empresas de nueva creación en Silicon Valley, mientras que los inmigrantes de China y Taiwán combinados fundaron el $12.8 \%$ (Chand y Ghorbani, 2011). Light resalta el hecho de que en el periodo de 1970-1999 los empresarios inmigrantes chinos y coreanos reorganizaron la morfología de Los Ángeles, California, construyendo nuevas comunidades para hospedar a sus paisanos migrantes. Y también en Europa, afirman Nijkamp, Sahin y Tuzin (2009), muchas ciudades se han convertido en sociedades pluriformes y multiculturales como resultado de la influencia estructural de los migrantes extranjeros en décadas recientes.

Es interesante resaltar lo que Vinogradov y Kolvereid (2010) refieren cuando analizan lo que ellos consideran la inteligencia de los empresarios migrantes. Ellos conciben la inteligencia como una capacidad mental, la cual implica entre otras cosas, la habilidad de razonar, planear, resolver problemas, pensar abstractamente, comprender ideas complejas, aprender rápidamente y aprender de la experiencia. Esta capacidad puede ser esencial para que los migrantes comiencen un nuevo negocio en el nuevo ambiente de la sociedad de destino. Empezar y manejar un negocio en un nuevo país requiere aprendizaje y adaptación mental y la habilidad para reconocer las oportunidades empresariales. Para Borjas (1986) la asimilación es importante para la construcción de un negocio de inmigrantes.

Es interesante hacer notar las aseveraciones de Bogan y Darity (2008) cuando hacen mención de las vetas culturales en el momento que tratan de explicar el éxito empresarial de los coreanos en Estados Unidos. Según estos autores los inmigrantes coreanos tienen los más altos índices de autoempleo entre los 17 grupos que llegaron a ese país entre 1970 a 1990. Tomando en cuenta la hipótesis culturalista esto puede ser explicado por sus atributos étniconacionales. Históricamente, un rasgo predominante de la cultura coreana fue la influencia de la civilización china especialmente a través del confusionismo, el cual ostensiblemente enfatiza el trabajo duro, la visualización al futuro, la movilidad social y las conexiones familiares. Aunado esto con sus antecedentes de clase media urbana que los provee de capital financiero inicial, formación universitaria y manejo de habilidades y actitudes.

\section{CONCLUSIONES}

El proceso de la migración internacional contemporánea ha adoptado una mayor complejidad en sus causas y consecuencias. Entre sus modalidades más destacadas tenemos un aumento muy notorio de los flujos de distintos tipos entre las fronteras que se manifiesta en la inversión, el comercio, los productos culturales, las ideas y las personas. Y la inversión de los migrantes en los países desarrollados ha crecido de manera importante reflejando ese, cada vez mayor, dinamismo del migrante en las sociedades de destino.

En el marco de la historia de la migración México-Estados Unidos, en las últimas décadas ha traído consigo un mayor volumen e intensidad del fenómeno, impactando de manera más significativa para el país. Asistimos, además del incremento de las remesas, al aumento de los empresarios migrantes mexicanos en Estados Unidos.

Al igual que las causas de la migración, los elementos propulsores de la 
formación del espíritu empresarial migrante ha sido objeto de reflexión desde hace muchas décadas, generando con ello varios enfoques teóricos. Unos enfatizan más las consideraciones macro (económicas, políticas, institucionales), otras se focalizan en las determinantes de tipo cultural, familiar, psicosocial. Y otras, más holísticas e integradoras, plantean la necesidad de analizarlo desde sus diferentes estructuras que observan desde lo macro a lo micro.

Sea como fuere, desde hace muchos años desde diferentes disciplinas, los científicos sociales han centrado su atención en el espíritu empresarial migrante. El recorrido que hemos realizado da cuenta de investigaciones teórica y empíricamente muy destacadas. La radiografía mostrada a través de indicadores como educación, estatus migratorio, lugares de destino, tradición empresarial familiar, elementos institucionales, capital humano y social, integración a la sociedad de destino, tradición nacional empresarial, entre otras importantes consideraciones, nos hacen considerar que el potencial del mexicano para invertir en Estados Unidos es grande y, en el futuro, es muy probable que se incremente.

Esto nos demuestra que hay que estudiar este fenómeno considerando dimensiones históricas, estructurales, coyunturales, regionales, de redes sociales, capital humano, social, simbólico, genética empresarial, tradición empresarial nacional, elementos informales e institucionales, de vínculos transnacionales, entre muchas otras.

Vaya encaminado este estudio a cumplir con dicho objetivo.

\section{BIBLIOGRAFÍA}

- Albo, Adolfo y Juan Luis Ordaz Díaz, "Migración mexicana altamente calificada a EEUU y transferencia de México a Estados Unidos a través del gasto en la educación de los migrantes", México, BBVA/Research, Documento de Trabajo, agosto 2011.

- Ariza, M., Portes, A. (2007). El país transnacional. Migración mexicana y cambio social a través de la frontera, México, UNAM-Instituto de Investigaciones Sociales.

- Assudani, R.H., "Ethnic entrepreneurship: The distinct role of ties", Journal of Small Business and Entrepreneurship, Vol. 22, No. 2, 2009, pp197-206.

- Azmat, Fara, "Exploring social responsibility of immigrant entrepreneurs: Do home country contextual factors play a role?" European Management Journal, Vol. 28, 2010, pp.337-386.

- BBVA/Research. (2012). Análisis Económico. Flash Migración México. Disponible en http://www.bbvaresearch.com/KETD/fbin/mult/120625 FlashMigracionMexico 13 tem346-335641.pdf?ts=2662012

- Basu, A., (1998), "An exploration of entrepreneurial activity among Asian small businesses in Britain", Small Business Economics, Vol.10, No.4, pp.313-326.

- Bogan, Vicki y William Darity Jr. (2008), "Culture and entrepreneurship? African American and immigrant self-employment in the United States", The Journal of Socio-Economics, Vol. 37, pp. 1999-2019.

- Borjas, G.J., (1986), "The Self-employment experience of immigrants", The Journal of Human Resources, Vol.21, pp. 485-506.

- Borjas, G.J., Katz, L.F. (2005). The Evolution of the Mexican-Born Workforce in the United States, Working Paper 11281. Disponible en http://www. nber.org/papers/w11281

- Borooah, V.K., y K. Hart, (1999), "Factors affecting self-employment among Indian and Black Caribbean men in Britain", Small Business Economics, Vol.13, No.2, pp. 111-129.

- Bovenkerk, Frank y Loes Ruland, (1992) "Artisan Entrepreneurs: Two Centuries of Italian Immigration to the Netherlands", International Migration Review, Vol. 22, No.3, pp.927-939.

- Cambio Climático, Degradación Ambiental y Migración, (2012), Suiza, Organización Internacional para las Migraciones (OIM).

- Castles, Sthephen, y Mark J. Miller, (2004), La era de la migración. 
Movimientos internacionales de población en el mundo moderno, México, Universidad Autónoma de Zacatecas-Miguel Ángel Porrúa.

- Cavusgil, Tamer, Dilek Zamantili Nayir, Gerd-Michael Hellstern, (editorial), (2011), "International Ethnic entrepreneurship", International Business Review, Vol.20, pp. 591-592.

- Cervantes, J.A. (2011). Remesas familiares y migración a Estados Unidos, México, Centro de Estudios Monetarios Latinoamericanos, Documento de Trabajo.

- Chand, Masud y Majid Ghorbani, (2011), "National culture, networks and ethnic entrepreneurship: A comparison of the Indian and Chinese immigrants in the US", International Business Review, Vol. 20, pp. 593-606.

- Chung Kim, Kwang y Won Mo Hurt, (1985) "Ethnic Resourses Utilization of Korean Immigrant Entrepreneurs in the Chicago Minority Area", International Migration Review, Vol. 19, No. 1, pp.82-111.

- CONAPO. (2010). Índices de Intensidad Migratoria México-Estados Unidos. Disponible en http://www.conapo.gob.mx/publicaciones/intensidad_ migratoria/texto/Migracion Mex EU.pdf

- Constant, Amelie, Yochanan Shachmurove y Klaus F. Zimmermann, (2007)"What Makes an Entrepreneur and Does it Pay? Native Men, Turks, and Other Migrants in Germany", International Migration, Vol.45, No.4, pp.71-100.

- Dai, Ou y Xiaohui Liu, (2009), "Returnee entrepreneurs and firm performance in Chinese high-technology industries", International Business Review, Vol.18, pp.373-386.

- Dai, Fu, Karen Yuan Wang y Stephen T.T. Teo, (2011), "Chinese immigrants in network marketing business in Western host country context", International Business Review, Vol.20, pp.659-669.

- Diaz, Mc Connell Eileen, (2008), "The U.S. Destinations of Contemporany Mexican Immigrants", International Migration Review, Vol. 42, No.4, pp. 767-802.

- Fairchild, Gregory B. (2010), "Intergenerational ethnic enclave influences on the likelihood of being self-employed", Journal of Business Venturing, Vol.25, pp.290-304.

- Fairlie, Robert W. y Alicia M. Robb, (2007), "Why Are Black-Owned Businesses Less Successful than White-Owned Businesses? The Role of Families, Inheritances, and Business Human Capital", Journal of Labor Economics, Vol. 25, No.2, pp. 289-323.
- Fairlie, Robert W., y Christopher Woodruff, (2007), "Mexican Entrepreneurship: A Comparison of Self-Employment in Mexico and the United States". http://www.nber.org/chapters/c0100

- Georgarakos, Dimitris y Konstantinos Tatsiramos, (2009), "Entrepreneurship and survival dynamics of immigrants to the U.S. and their descendants", Labour Economics, Vol.16, pp.161-170.

- Gonzalez-Gonzalez, José María, Francisco D. Bretones, Victoria Zarco y Andrés Rodriguez, (2011), "Women, immigrationand entrepreneurship in Spain: A confluence of debates in the face of a complex reality", Women's Studies International Forum, Vol.34, pp.360-370

- Homeland Security (U.S. Naturalizations: 2011). http://www.dhs.gov/ xlibrary/assets/statistics/publications/natz_fr_2011.pdf

- Ibrahim, Gamal y Vaughan Galt, (2011), "Explaising ethnic entrepreneurship: An evolutionary economics approach", International Business Review, Vol.20, pp.607-613.

- Ilhan-Nas, Tulay, Kader Sahin y Zuhal Cilingir, (2011), "International ethnic entrepreneurship: Antecedents, outcomes and environmental context", International Business Review, Vol.20, pp. 614-624.

- INEGI. Disponible en http://www.inegi.org.mx

- IOM, (2012). International Organization for Migration. Disponible en http:// www.iom.int/jahia/jahia/factors-and-figures/lang/es

- Kauffman Foundation (2009). Kauffman Index of entrepreneurial activity. http://www.kauffman.org/uploadedFiles/kiea_042709.pdf

- Kesler, Christel y Michael Hout, (2010), "Entrepreneurship and immigrant wages in US labor markets: A multi-level approach", SocialScience Research, Vol.29, pp.187-201.

- Ley, David, (2003), "Seeking Homo Economicus: The Canadian State and the Strange Story of the Business Immigration Program", Annals of the Association of American Geographers, Vol. 93, No. 2, pp.426-441.

- Li, P., (2001), "Immigrants propensity to self-employment: evidence from Canada", International Migration Review, Vol.35, No.4, pp.1106-1128.

- Light, Ivan, (2002), "Immigrant Place Entrepreneurs in Los Angeles, 197099", International Journal of Urban and Regional Research, Vol.26, No.2, pp.215-228.

- McLeman, Robert, (2012), Climate change, migration and critical international security considerations, Suiza. International Organization for Migration/Migration Research Series. 
- Nijkamp, Peter, Mediha Sahin y Tüzin Baycan-Levent, (2009), “Migrant Entrepreneurship and New Urban Economic Opportunities: Identification of Critical Success Factors by Means of Qualitative Pattern Recognition Analysis", Tijdschrift voor Economische en Sociale Geografie, Vol.00, No.0, pp.000-000.

- Pew Hispanic Center. Disponible en http://www.pewhispanic.org

- Pio, Edwina, (2007), "Ethnic Entrepreneurship among Indian Women in New Zealand: A Bitters-weet Process", Gender, Work and Organization, Vol.14, No.5, pp.409-432.

- Portes, Alejandro, Luis Eduardo Guarnizo y William J. Haller, (2002), "Transnational Entrepreneurs: An Alternative Form of Imigrant Economic Adaptation", American Sociological Review, Vol.67, No.2, pp.278-298.

- Portes, A., y M. Zhou, (1996), "Self-employment and the earnings of immigrants", American Sociological Review, Vol. 61, pp.219-230.

- Raijman, Rebeca, (2001), "Determinants of entrepreneurial intentions: Mexican immigrants in Chicago", Journal of Socio-Economics, Vol.30, No.5, pp.393-411.

- Riddle, Liesl y Jennifer Brinkerhoff, (2011), "Diaspora entrepreneurs as institutional change agents: The case of Thamel.com", International Business Review, Vol.20, pp.670-680.

- Riddle, Liesl, George A. Hrivnak, Tjai M. Nielsen, (2010), “Transnational Diaspora entrepreneurship in emerging markets: Bridging institutional divides", Journal of International Management, Vol. 16, pp.398-411.

- Robles, Bárbara J., y Héctor Cordero-Guzmán,(2007), "Latino SelfEmployment and Entrepreneurship in the United States: An Overview of Literature and Data Sources", The ANNALS of the American Academy of Political and Social Science, Vol. 613, No.1, pp.18-31.

- Shinnar, Rachel S., et-al, (2009), "Immigrant and US-Born Mexican-Owned Businesses: Motivations and Management", Journal of Developmental Entrepreneurship, Vol.14, No.3, pp.273-295.

- Taylor, Paul S., (1930), "Some Aspects of Mexican Immigration", The Journal of Political Economy, Vol. 38, No. 5, 1930, pp.609-615.

- Vinogradov, Evgueni y Lars Kolvereid, (2010), "Home country national intelligence and self-employment rates among immigrants in Norway", Intelligence, Vol.38, pp.151-159.

- Yang, Chulguen, Stephen M. Colarelli, Kyunghee Han, Robert Page, (2011), "Start-up and hiring practices of immigrant entrepreneurs: An empirical study from an evolutionary psychological perspective", International Business Review, Vol.20, pp.636-645.

- Yuengert, M.A., (1995), "Testing hypotheses of immigrant self-employment", Journal of Human Recourses, Vol.30, No.1, pp.194-204.

Dr. Eduardo Fernández Guzmán.

Miembro del Sistema Nacional de Investigadores, Nivel 1. Licenciado y Maestro en Historia por la Universidad Michoacana de San Nicolás de Hidalgo de Morelia, México. Doctor en Historia Moderna y Contemporánea en el Instituto Mora (mención honorífica). En la actualidad es profesor-investigador de tiempo completo del Departamento de Estudios Culturales, Demográficos y Políticos de la División de Ciencias Sociales y Administrativas de la Universidad de Guanajuato Campus Celaya-Salvatierra. Además, es miembro de la Red Internacional de Migración y Desarrollo. Sus líneas de investigación son la historia contemporánea de la migración internacional México-Estados Unidos, así como las causas y consecuencias de la migración de retorno en las comunidades de origen.

Email: kutibirrin10@gmail.com

Dra. Perla Shiomara del Carpio Ovando.

Doctora y maestra en Psicología Social por la Facultad de Ciencias Políticas y Sociología de la Universidad Complutense de Madrid. Licenciada en Psicología por la Universidad de Ciencias y Artes de Chiapas. Ha participado en congresos nacionales e internacionales y ha sido acreedora de diversos premios entre los que sobresale el Premio Especial otorgado por la Sociedad Española de Psicología Experimental en el III Certamen Teresa Pinillos de Divulgación Científica (2012), 
el Primer Lugar otorgado por la Asociación Castellano Manchega de Sociología en el concurso de Ensayo Breve Fermín Caballero (2009), el Premio Estatal de la Juventud (2009), otorgado por el Gobierno del Estado de Chiapas y el Premio en redacción Carta a mis padres (2011), otorgado por el Instituto Mexicano de la Juventud. Actualmente es profesora e investigadora de tiempo completo en la Universidad de Guanajuato, Campus Celaya-Salvatierra, División de Ciencias Sociales y Administrativas, Departamento de Estudios Sociales. Sus líneas de investigación actuales son: identidad, trabajo, artesanías, pueblos indígenas y procesos migratorios.

Email: pdelcarpio@ugto.com

Dr. Eloy Mosqueda Tapia

Doctor en Ciencia Social con Especialidad en Sociología por El Colegio de México. Maestro en Ciencias Sociales por la Facultad Latinoamericana de Ciencias Sociales. Licenciado en Sociología por la UNAM, Facultad de Estudios Superiores Aragón. Profesor de Tiempo Completo en la Universidad de Guanajuato, desde enero de 2013. Impartiendo cursos y seminarios en las carreras de Maestría en Fiscal, Mercadotecnia, Administración Financiera y Tronco Común. Profesor a nivel superior en la Licenciatura en Sociología, UNAM, FES Aragón, desde 1999 a 2012, en cursos y seminarios que abarcan desde la teoría social hasta la metodología.

Email: eloymosta@yahoo.com.mx 